\title{
Antibiotics in food and environmental samples
}

\author{
María Cruz Moreno-Bondi
}

Published online: 16 August 2009

(C) Springer-Verlag 2009

Since the discovery of penicillin by Alexander Fleming in 1928, antibiotics have revolutionized the treatment of infectious diseases, contributing significantly to improving the health of the population, decreasing morbidity and mortality. The first antibiotics to be applied in human and veterinary medicine were of natural origin, produced by fungi or bacteria. Nowadays, they are also obtained by chemical synthesis or by chemical modification of compounds of natural origin, and currently the term 'antibiotic' refers to any substance with antibacterial, antifungal or antiparasitic activity of natural, synthetic or semisynthetic origin.

There are about 250 different chemical substances registered for use in human and veterinary medicine, although the usage patterns differ from one country to another $[1,2]$. The total global antibiotic consumption was estimated to be between 100,000 and 200,000 tons per annum. According to a survey carried out in 2000 by the European Federation of Animal Health (FEDESA), 13,216 tons of antibiotics were used in the EU and Switzerland in $1999,65 \%$ of which were applied in human medicine, $29 \%$ in veterinary medicine and $6 \%$ as growth promoters. Between 1997 and 1999, the total antibiotic consumption increased by about $10 \%$, although the use of antibiotics as growth promoters decreased by $51 \%$ in this period. However, these numbers refer only to prescribed antibiotics, but in some countries self-diagnosis and medication are a common practice. On the other hand, according to a recent report, in the USA approximately 11,200 metric tons

M. C. Moreno-Bondi $(\bowtie)$

Optical Chemosensors and Applied Photochemistry Group (GSOLFA), Department of Analytical Chemistry,

Faculty of Chemistry, Universidad Complutense de Madrid, 28040 Madrid, Spain

e-mail: mcmbondi@quim.ucm.es of antibiotics were used as growth promoters for cattle, hogs and poultry [1].

Antibiotics are also used in veterinary medicine and in aquaculture, for the prevention (prophylaxis) and treatment of microbial infections. They may also be added to animal feeds at low (subtherapeutic) levels to improve the growth rate and efficiency of feed utilization, reduce mortality and morbidity and improve reproductive performance [3]. In this regard, since 1 January 2006 the European Union has banned the use of these drugs as growth promoters in animal feed [4]. In addition, antibiotics are also used in agriculture, mainly for the control of bacterial diseases of tree fruits and ornamental plants [1], affecting other food producers such as honeybees.

One of the main drawbacks associated with antibiotic usage is the development of antimicrobial resistance. There is a global resurgence of infectious diseases occasioned by new infectious agents, as well as by the re-emergence of the antibiotic-resistant old ones. The appearance and evolution of microbial resistance is a serious clinical and public health problem on a global basis that threatens human and animal health alike [5]. Drug choices are becoming increasingly limited as investments in the development of new antimicrobials to address the global problems of drug resistance are scarce. Features such as the transfer of resistance from food-producing animals to humans via the food chain, or the development of multiresistance and the ability to transfer this resistance to other organisms, have given rise to widespread international debate. As a consequence, there is increasing interest in policies and guidelines to reduce antibiotic use and to improve the quality of prescribing antibiotics and consequently the patient outcome. Important multimedia campaigns have been launched by international organizations and national authorities among physicians, public health experts, con- 
sumers and producers, to promote the rational and controlled use of antibiotics in humans and foodproducing animals to contain antibiotic resistance $[6,7]$. The World Health Organization has published a global strategy for containment of antimicrobial resistance to provide a framework for interventions to slow down the emergence and reduce the spread of antimicrobial-resistant microorganisms. The strategy includes among other recommendations (1) a reduction in the disease burden and the spread of infection, (2) fostering access to appropriate antimicrobials for each treatment, (3) improving the use of antimicrobials, avoiding overuse, particularly for minor infections, or underuse due to lack of financial support to complete treatment courses, (4) strengthening health systems and their surveillance capabilities, (5) enforcing regulations and legislation and (6) encouraging investment in the development of new drugs and vaccines [8]. Regulatory agencies in the EU and the USA have issued detailed guidelines on the assessment of veterinary and human drugs to avoid unwanted effects in the environment [9-11].

Antibiotics are included in the group of pharmaceutically active compounds (PhACs) considered as environmental emerging contaminants [12]. These drugs, in comparison with conventional priority pollutants, will have a specific pharmacological and physiological effect, even at low doses. On the other hand, in contrast to other PhACs, their concentration in influent loads may be different in winter and summer [13]. Therefore, issues related to their occurrence, fate, transport or degradation in the environment have become a serious concern for the scientific community.

This special issue of Analytical and Bioanalytical Chemistry presents an overview on the state of the art of antibiotics analysis in food and environmental samples. Current research in this field is quite broad and challenging and requires the joint effort of experts in different areas, such as chemistry, biology, health, the veterinary science and ecotoxicology, to address the important issues raised above. This themed issue covers some important aspects related to current analytical methods applied to antimicrobial analysis, legislation and selected applications. I would like to thank all the authors for their excellent contributions that demonstrate the great impact of and interest in antibiotics in our daily life.

\section{References}

1. Kümerer K (2008) J Antimicrob Chemother 52:5-7

2. ESAC (2009) European surveillance of antimicrobial consumption. http://www.esac.ua.ac.be/

3. Cromwell GL (2002) Anim Biotechnol 13:7-27

4. European Commission (2003) Regulation 1831/2003/EC on additives for use in animal nutrition, replacing Directive 70/524/ EEC on additives in feeding-stuffs

5. Lord Soulsby of Swaffham Prior (2008) J Antimicrob Chemother 62:229-233

6. Goossens H, Coenen S, Costers M, De Corte S, De Sutter A, Gordts B, Laurier L, Struelen MJ (2008) Eurosurvellance 13:1-4

7. WHO (2002) Antimicrobial resistance. http://www.who.int/mediacentre/factsheets/fs194/en/

8. WHO (2001) WHO global strategy for containment of antimicrobial resistance. executive summary. WHO, Geneva

9. EMEA (1998) Note for guidance: environmental risk assessment for veterinary medicinal products other than GMO-containing and immunological products. EMEA/CVMP/055/96. EMEA, London

10. EMEA (2005) Note for guidance: on environmental risk assessment of medicinal products for human use, CMP/SWP/444/draft. The European Agency for the Evaluation of Medicinal Products, EMEA, London

11. FDA-CDER (1998) Guidance for industry environmental assessment of human drugs and biologics applications, revision 1. FDA, Centre for Drug Evaluation and Research, Rockville

12. Rodríguez-Moraz S, López de Alda MJ, Barceló D (2007) J Chromatogr A 1152:97-115

13. Barceló D, Petrivic M (eds) (2008) Emerging contaminants from industrial and municipal waste. Occurrence, analysis and effects. Springer, Berlin

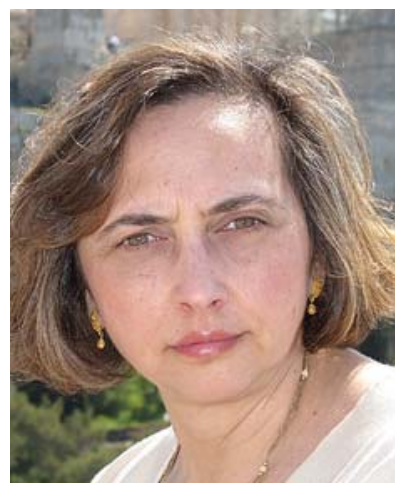

María C. Moreno-Bondi is Professor of Analytical Chemistry at Complutense University in Madrid (Spain). Her main areas of research include the development of optical chemical sensors and biosensors based on fluorescence measurements and their application to environmental and food analysis, and the synthesis of biomimetic recognition elements for sensing and separation purposes. She received the Young Researcher's Award from the Spanish Society of Analytical Chemistry in 1993. She is a member of the executive boards of the Spanish Society of Applied Spectroscopy, the Madrid Community Section of the Royal Spanish Society of Chemistry and also of the Advanced Study Course on Optical Sensors (ASCOS) series. 